\section{TRAUMA CARE}

\section{Trauma care-a vision for the future}

For want of trauma surgery as a specialty in the UK, the management of major trauma has come to be seen as the responsibility of specialists in accident and emergency (A\&E) medicine. The management of major trauma perhaps attracts more trainees than any other aspect of the specialty. Consultants all over the country struggle to establish multidisciplinary "trauma teams" and the competition for Advanced Trauma Life Support (ATLS) course places leads to long waiting lists. Yet despite all this interest and activity, the management of the victim of trauma remains suboptimal in many hospitals.

Perhaps the main reason for this is lack of communication between specialties. We have all sat in coffee rooms at antisocial hours and bemoaned the attitude of one or other specialty regarding their response to a particular patient. Yet in many centres, and indeed nationally, there is no culture of specialties training together, learning together, and working together as equals to improve the outcome for trauma patients. It is very easy for specialties, each recognised as excellent in their own field, to contribute to suboptimal overall management when working together.

Apart from promoting the best evidence based resuscitation, $A \& E$ specialists are ideally placed to promote integrated trauma care. As we have all realised, the optimal management of a patient is more than the optimal management of a patient's individual injuries.

The organisation "Trauma Care" was founded in 1996 to promote just such integrated trauma care. After a successful launch conference in Harrogate in 1997, which was designed to promote the philosophy of integrated, multidisciplinary care, the organisation has grown and now represents all the medical specialties involved in trauma care as well as members of specialties allied to medicine such as nurses, paramedics, and scientists.

The abstracts published below are from our most recent conference in Bournemouth. Four hundred delegates attended and the conference was designed to encourage doctors, nurses, and paramedics to participate. The organisation continues to work towards establishing and promoting multispecialty, multidisciplinary evidence based best practice.

The review journal Trauma was launched successfully in 1998 and, in 2000, Trauma Care expert working groups will issue evidence based guidelines for best practice in a number of clinical areas. Also in 2000, the conference Trauma 2000 will see the launch of a new manual of trauma care based on the best of European practice.

Trauma Care is currently the only organisation working in the field of trauma that truly represents all specialties and disciplines, and we are delighted that interest in setting up a similar body has been expressed by leading trauma clinicians in a number of other countries. Our sister organisation Trauma Care (South Africa) is currently being established.

In the UK, we intend to develop a wide range of initiatives including educational projects and the promotion of research and best practice. A research and development committee has recently been formed. We will in due course be moving from a committee based organisation to an open membership with Trauma as our society journal.

May I encourage you to join us in London for Trauma 2000, and to contribute to Trauma Care. The future of this organisation is bright and the opportunities great but in order to work towards the best possible outcome for trauma victims in this country and abroad, we need your support. If you would like more information about the London conference, please contact the Conference Secretariat at Index Communications Meetings Services, Crown House, 28 Winchester Road, Romsey, Hampshire SO51 8AA.

IAN GREAVES

Consultant in Emergency Medicine, British Army, Accident and Emergency Department, Peterborough District Hospital, Thorpe Road, Peterborough PE3 6DA

\section{ABSTRACTS}

\section{2nd Trauma Care Conference: Improving Trauma Care, Bournemouth, 7-9 June 1999}

\section{Oral presentations}

Amputations at the Royal London Hospital 1852-57

\section{E Chaloner}

Leonard Cheshire Department of Conflict Recovery, University College, London

A retrospective analysis of amputations from the archive records of the Royal London Hospital, Whitechapel.

During the period studied, amputations accounted for $34 \%(142 / 400)$ of all operations performed in the hospital. There were 44 upper limb ( 25 above and 19 below elbow) and 98 lower limb amputations ( 51 above and 47 below knee) on 121 males (mean age 29 years; range 1-60) and 15 females with a mean age of 31 years (range 2-75). Six men had bilateral lower limb amputations.

Trauma accounted for 35 arm and $50 \mathrm{leg}$ amputations $(60 \%$ of the total). Industrial accidents caused 27 out of 72 incidents where the cause of accident was known. Sailors, labourers, and railway workers were especially prone to falling victim to accidents requiring amputation.

The overall mortality was approximately $50 \%$ (61 deaths, 69 survivors, six unknown). Of the lower limb amputations carried out for trauma, 34 patients died and 16 survived. Of the upper limb amputations, eight died and 28 survived.

The cause of death (where recorded) was commonly shock (11 cases) or sepsis (32 cases). Chloroform was used in 30 of 136 cases. The mortality where chloroform was used for trauma cases was higher (11 deaths out of 17 cases) than in trauma cases where it was not used ( 23 deaths from 53 cases).

The above data illustrate the high mortality of amputation in the 19 th century. Compared with other contemporary military and civilian figures the mortality is quite high, especially the combination of chloroform and trauma. This may reflect the poor nutritional state of patients in the East End or the effects of anaesthetising patients who were inadequately resuscitated.
The lived experience of resuscitation for accident and emergency nurses $\mathrm{C}$ Williams

Accident and Emergency, West Wales General Hospital, Carmarthenshire

A phenomenological method was used to describe the lived experience of resuscitation for accident and emergency nurses. Twelve nurses were purposefully selected after taking part in a resuscitation. Data was collected from two unstructured interviews with each participant, and then analysed using Colaizzi's (1978) methodology. Findings were written into an exhaustive description of the lived experience, and then returned to the participants for validation.

The lived experience of resuscitation was found to be occasionally positive, producing feelings of achievement and pleasure, due on most occasions to perceptions of teamwork and support from competent colleagues. On other occasions the lived experience engendered feelings of anxiety, distress, frustration, horror, sadness, and anger caused by organisational factors as well as those intrinsic to nursing critically ill patients. Death, if it occurred, was found to come with a period of unacknowledged awareness before a consensus decision was made to end the resuscitation. Death that was perceived as inevitable caused feelings of sadness; death that was unexpected or unaccepted caused intense distress. Talking to relatives after the death was associated with feelings of acute anxiety.

Many staff displayed some signs of stress after a resuscitation, but good social support systems within the workplace enabled the experience, although not forgotten, to be accepted and integrated into the nurses' life experiences within a short period of time.

Recommendations were made to improve organisational factors within resuscitations, increase education for staff in the care of bereaved relatives, and to encourage and sustain the informal social support system already in place.

\section{Emergency management of toxic trauma} D Baker

SAMU 75, Paris, France

Toxic trauma may be defined as the effects on casualties after the accidental or deliberate release of toxic substances. Such a release may occur in both peace and war and may be unexpected. Emergency medical responders should therefore be aware of the problems of toxic release, of the dangers of working in a contaminated environment, and of the special applications of site safety required. They should also be aware of the immediate measures necessary for effective medical management.

Toxic agents cause multisystem dysfunction but the effects on the respiratory system may be life threatening. Recent developments in equipment and training allow the provision of protocols for the provision of advanced life support for toxic trauma within a contaminated environment (TOXALS) and can overcome potentially life threatening delays in the provision of medical care.

Ventilatory support in toxic trauma is vital and is now available using a portable stand alone automatic ventilator, developed originally from a military specification, and which has been produced for both military and civil use. The rationale and practicalities of the use of advanced life support equipment in a toxic zone will be presented within the framework 
of the United Nations hazardous materials convention (HAZMAT).

Accident and emergency nurses' perceptions of the effect of trauma training in clinical practice

L Jenkins

Accident and Emergency, West Wales General Hospital, Carmarthenshire

An exploratory descriptive design was used to explore the perceptions of the effect of trauma training. Twelve accident and emergency (A\&E) nurses were purposefully selected from an $A \& E$ department comprising 44 qualified staff. A critical incident technique incorporating semistructured interviews was used to collect data, which was then analysed using content analysis. Four categories emerged: knowledge and skills, perceptions of "self" in the trauma room, promotion of teamwork, and emergence of the trauma trained nurse.

Trauma resuscitation was described as a stressful event that engendered feelings of being overwhelmed. Training was perceived to have increased knowledge, skills and confidence levels, contributing to improvements in injury recognition and effective communication. Perceptions of an ability to positively influence patient care and outcome were associated with these findings. Occasional feelings of frustration were caused by failure to follow a systematic approach and perceptions of physician failure to act on concerns expressed by the nurse. Effective role performance and teamwork were seen as a result of undertaking a joint training programme. In the initial stages, the emergence of trauma trained nurses caused feelings of inadequacy among those nurses who had not yet been trained. This resulted in a degree of staff conflict which diminished as more nurses became trauma trained and visible benefits were observed.

Recommendations are made that nurses involved in trauma care should be educated following the universally recognised protocols of the Advanced Trauma Life Support course and that they should participate in joint learning programmes.

Expertise in trauma care? Get it recognised

V Heath

University of Plymouth, Faculty of Human Sciences, Institute of Health Studies

One of the outcomes of successful trauma care is the recognition of clinical expertise. Expertise in practice is, however, very much a shared multi/interdisciplinary outcome. So what of the individual trauma nurse who plays a specific, but essential role. How does that individual gain recognition on an individual basis? Certainly one's self esteem elevates considerably, but how can one gain recognition that will support you in your aim to provide evidence for your own personal and professional development purposes?

The flexibility offered through Accreditation of Prior (Experiential) Learning $(A P(E) L)$ encourages both professional and personal growth through presentation of clinical experience as evidence of value. This is clearly a critical factor at a time when declining resources (particularly that of time), present practitioners and employers with considerable difficulties in providing opportunities for professional development. The knowledge that practical expertise in trauma care will be recognised as equal to academic award by the employer and the provider of nurse education can only auger well for all.

The way forward for your clinical expertise to be recognised ? $\mathrm{AP}(\mathrm{E}) \mathrm{L}$.

Are blood samples obtained from bone marrow useful for blood analysis?

J Hurren

Wessex Regional Burns Unit, Salisbury

Introduction-Under circumstances of vascular collapse intraosseous cannulation provides an important second line access to the circulation for resuscitation in children. It would clearly be valuable if once the cannula was placed blood could be drawn off for haematological and biochemical analysis. The aim of this study was to investigate the potential accuracy of analysis of such samples.

Method-Fourteen adult patients undergoing routine bone marrow aspiration as a haematological investigation were investigated. Ethical committee approval and patient consent was obtained. Blood aspirated from bone marrow was compared with two blood samples taken from a peripheral vein by routine laboratory analysis.

Results-Haemoglobin, sodium, urea, creatinine, glucose, and calcium concentrations were found to be similar in samples from the bone marrow and peripheral venous blood. Observed differences were so small they would be unlikely to be of clinical consequence. The value for potassium was consistently higher in bone marrow samples compared with peripheral venous blood. However the $95 \%$ confidence interval was -0.002 to $0.510 \mathrm{mmol} / 1$. This difference is sufficiently small so as to still give a clinically useful indication of the true serum potassium concentration. The values for white cell count and platelet count were very different between bone marrow and peripheral venous samples. Two of the aspirated samples were very fatty and unsuitable for analysis.

Conclusion-This study suggests that blood samples taken from the bone marrow can be analysed to provide clinically useful measurements of the principal blood and serum parameters that are required in the initial stages of resuscitation.

Delayed diagnosis of odontoid peg fractures

N Ashwood, J Parfait, P Hallam, J Goodall Central Middlesex Hospital, London

Odontoid peg fractures generally occur during high energy injuries in association with head injuries and it is estimated that $40 \%$ of these fractures are missed in the early post-injury period. Our experience identifies a large subgroup of patients who sustained the same fracture after minor trauma and in whom the diagnosis is often delayed.

We prospectively identified 12 patients presenting to the accident and emergency (A\&E) department at our institution with a final diagnosis of odontoid peg fracture. There were seven patients who had initially been diagnosed with minor injuries to the neck, four after assaults and the remaining three from falls from less than one metre. All seven reattended an average of 4.3 weeks after their injury, three via the radiology department after radiography organised by the general practitioner and four via the A\&E department. Clinically the patients noted continuing neck pain and limited range of motion. There was significant cervicodorsal kyphosis in two cases, but no patients in the series had neuro- logical impairment. Treatment involved surgical stabilisation in two cases with transpedicular screws, three were managed in halo braces for 4-6 weeks, and the remainder were treated conservatively. We contacted $20 \mathrm{~A} \& \mathrm{E}$ consultants, $12(60 \%)$ of whom reported a missed odontoid peg fracture in their department within the last year.

In conclusion, a high index of suspicion is necessary to diagnose odontoid peg fractures. The mechanism of injury can be trivial, history vague, and presentation delayed.

Day surgery hand trauma: preliminary experience at the Queen Victoria Hospital

J Garner, F Schonauer, J Pereira, M Pickford Queen Victoria Hospital, East Grinstead, West Sussex

Trauma is now responsible for $40 \%$ of the workload carried out by the plastic surgery department and the vast majority of trauma cases are upper limb injuries. Many of these are complex and require admission and inpatient treatment, but a sizeable proportion of these injuries can be reasonably managed on an outpatient basis. In order to utilise resources more efficiently and provide a better service for patients, we introduced a system whereby appropriate hand injuries were managed on a day case basis. This study was designed to assess the impact of this system.

The case notes of the first 66 hand trauma patients managed as outpatients at the Day Surgery Unit between November 1998 and January 1999 were retrospectively reviewed with emphasis on type of injury, surgery performed, and clinical outcome.

The commonest procedures performed were isolated digital nerve repair (13/66) and repair of the flexor digitorum profundus tendon (9/66). Regional or local anaesthetic techniques were used in 64 patients. Two patients required general anaesthetic. No patients required admission to hospital.

The introduction of a day surgery hand trauma operating list has great potential benefits for both patients and the hospital. The patients receive their treatment in a timely manner with early involvement of the hand therapists. Inpatient beds are used more effectively for elective surgery cases. Moreover this system has enabled consultant supervision of the majority of hand trauma surgery.

Perioperative blood transfusion in femoral neck fractures-too much transfusion? O Odumala, C Ayekoloye, G Packer Southend Hospital NHS Trust, Essex

Objective-To identify factors that determine blood transfusion and analyse if they were appropriately transfused, based on the guidelines of the American College of Physicians (ACP).

Methods-A prospective longitudinal study of 110 consecutive patients operated on for fractured neck of femur. Patients were assessed for the risk of myocardial or cerebral ischaemia as outlined by the ACP guidelines. Weighing swabs and measuring the drainage volume in the suction apparatus assessed operative blood loss.

Results-The study population comprised of 20 men and 90 women; mean (SD) age was $81.6(12.3)$ years. Over one third of patients $(34.5 \%)$ had blood transfusion. Significant variables associated with blood transfusion were the preoperative haemoglobin, postoperative haemoglobin, and postoperative blood 
loss $(p<0.05)$. Patients at risk and American Society of Anaesthesiologists (ASA) class III and IV patients were given more blood but it was not statistically significant $(p>0.05)$.

Thirty eight patients were transfused with a total of 92 units of homologous SAM-M blood of which 56 units $(63 \%)$ were inappropriate. Seventeen units $(18 \%)$ were given perioperatively in haemodynamically stable patients and 39 units (45\%) transfused in asymptomatic patients to achieve a haemoglobin concentration of $100 \mathrm{~g} / 1(10 \mathrm{~g} \%)$. High risk patients, patients in ASA classes III and IV, and blood loss were significantly higher in appropriately transfused patients. $(p<0.05)$. The preoperative and postoperative haemoglobin and mortality were similar in both groups $(p>0.05)$.

Conclusion-There is need to review blood transfusion practices and the emphasis on haemoglobin concentrations discouraged. The ACP guidelines will reduce unnecessary transfusions.

\section{Sensory neuropeptide in orthopaedic pa-} tients

G N Onuoha, E K Alpar

University of Birmingham

An intact nociceptor system of primary afferent sensory nerves is important for the initiation of the inflammatory process and successful tissue repair. Dysfunction of this system with accident or trauma could be a contributing factor for the delayed wound healing in orthopaedic patients. The ability of the sensory peptides in modulating wound healing is known by taking into account the modulatory interaction effects between the peptides. This study was designed to examine the release mechanism of these wound modulatory peptides in patients with long bone fractures. We have measured the peripheral plasma concentrations of sensory regulatory peptides (calcitonin gene related peptide (CGRP) and substance P) immunoreactivity in orthopaedic patients (mean age 70, range 20-90 years, $n=18$ ) within two days of injury and compared with healthy control subjects (mean age 39.2, range $17-91$ years, $n=17$ ) Neuropeptides were measured by enzyme immunoassay while elastase, creatine kinase, and myoglobin were measured by ELISA techniques.

Mean (SD) ng/l of CGRP was higher in patients both in day 1 (309.7 (195.6)) and day 2 (184.4 (161.2)) compared with controls (65.8 (26.9)); $p<0.05$. Substance $P$ also increased in the patients (day 1, 63.9 (43.8), day $2,47.0(7.2)$ ) compared with controls (8.1 $(4.0)) ; \mathrm{p}<0.05$. Furthermore, elastase (a predictor of post-traumatic complication), was examined and found to be higher in patients in both days of injury (day $1,192.6$ (126.2); day 2, 162.9 (140.6)) than controls (95.8 (32.6)) but the different in release within groups was not significant. Myofibrillar proteins were accordingly higher in patients than the control subjects; however the release of the proteins were higher in the second day of the study than the day of admission.

This study shows that sensory nerve peptides are present and increased in fracture related injuries, an increased level that is maintained 24 hours after hospital admission. In comparison with healthy controls this study also suggests that the release mechanism of substance $P$ was slightly higher than CGRP in the peripheral circulation of these orthopaedic patients. However the clearance rate of the two peptides from the circulation were about the same. This release mechanism could be of significant in the inflammatory and healing processes observed in this group of patients.

\section{The pre-burns unit management of the} burnt patient

H Ashworth, T Cubison

Queen Victoria Hospital, East Grinstead, West Sussex

Objectives-To review the accuracy of burn percentage assessment in the casualty department, the adequacy of fluid resuscitation and analgesia, the timing of transfer to a specialist burns unit and the transfer documentation.

Method -A retrospective analysis of the notes of all the UK patients admitted to the burns unit between January 1998 and July 1998, who had a body surface area (BSA) burn of over $15 \%$ in adults or $10 \%$ in children.

Results-There were 14 patients, seven adults and seven children; seven were male and seven female and the average burn size was $26 \%$ $(52 \%-12 \%)$. Six patients had their burn underestimated by average of $8.25 \%$ BSA, seven patients were overestimated by an average of $8.5 \%$ BSA. Twelve patients had intravenous fluids. In six patients the formula used was documented, but only in three was it used correctly. The fluid volumes given and urine output were poorly documented. Twelve patients received opiates, one by infusion, seven with intravenous boluses, three intramuscularly, and one orally.

The average time to the burns unit from the burn was 11.6 hours, to the referring hospital 8.3 hours, and the average time taken for resuscitation and transfer was 4.5 hours. Nine patients had letters of referral either as a letter or simply a burn proforma.

Conclusions-There is considerable variation in the standard of initial burn management and there may be a role for a new burn proforma to improve transfer documentation and provide an immediate source of referrence.

Diagnosis and management of acute compartment syndrome. A two year review of one hospital's experience

W J Hart, N Reynolds

Frenchay Hospital, Bristol

We reviewed 60 patients with compartment syndrome treated at Frenchay Hospital over a two year period. The review includes the mechanism of injury, fracture patterns, demographic details, and onset of symptoms. We go on to review the method of diagnosis, the use of intracompartmental monitoring, and the placement of fasciotomy incisions. In the postoperative period we look at techniques for reconstruction and direct closure. All patients have had at least 12 months of follow up and assessment of their functional outcome. The paper concludes by looking at the incidence of compartment syndrome with respect to fracture location, the age of the patient, and the mechanism of injury.

An analgesic protocol for military and civilian disaster relief operations G Hocking, W F DeMello*

Royal Devon and Exeter Hospital, Exeter, Devon

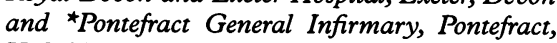
Yorkshire

We previously designed an analgesic protocol to improve the efficacy and safety of opioid based analgesia for the injured serviceman in the absence of medical expertise. ${ }^{1}$ We now present an advanced version of the analgesia protocol for use both in military and civilian disaster relief operations (fig 1 , see next page). Our aim was to provide the maximum analgesic benefit for the maximum number of patients using the minimum drugs and personnel. Therefore we considered factors such as drug availability, expertise of the personnel, logistic constraints, and the aetiology and chronological evolution of the pain. Analgesia is only administered to those patients who have cardiorespiratory stability, are alert or verbally responsive, and say they are in pain. If the cause of the pain is known the treatment is based on the VAS and its trends. The analgesic ladder has three choices ranging from enteral non-opioid drugs to intravenous opioids. Non-steroidal antiinflammatory drugs are considered if cardiorespiratory stability is attained. Antiemetics and antiemetic rotation is also included. Our choice of drugs are based on those available to British forces in Bosnia; however alternative drugs could equally be used using the same algorithic protocol

We feel that this protocol offers a framework for the safe administration of analgesia to patients in pain in either military or civilian disaster relief operations.

1 Hocking G, DeMello WF. $\mathcal{f} R$ Army Med Corps 1996;142:82-3.

Management of isolated sternal fractures J R Sadaba, D Oswal, C M Munsch

Yorkshire Heart Centre, Leeds General Infirmary, Leeds

Objective-To determine the risk of blunt cardiac injury in isolated sternal fractures.

Method-(1) Review of hospital records of 37 consecutive patients admitted to a cardiothoracic unit, with the diagnosis of isolated sternal fracture, from April 1995 to March 1997 and those of the six patients admitted with the same diagnosis, between September 1997 and September 1998. (2) Analysis of questionnaires sent to 27 cardiothoracic units in the UK on the management of isolated sternal fractures.

Results-(1) Electrocardiography (ECG) was performed in all 37 patients and showed no abnormality in 26 . Twenty nine patients underwent echocardiography, which failed to demonstrate significant effusion in all cases. Out of 10 patients who had creatine kinase levels tested, five had values above $140 \mathrm{IU} / 1$. Only one patient developed a complication in the form of atrial fibrillation. Based on recent reports and on our findings we introduced new guidelines for the management of isolated sternal fracture by which patients presenting with this condition, who are otherwise fit and well and who have normal ECG and chest radiography, are discharged home. After this, only six patients with isolated sternal fracture were admitted over a 12 month period. (2) Management of isolated sternal fractures in cardiothoracic units in the UK is quite heterogeneous.

Conclusion-Patients presenting with isolated sternal fracture and who are otherwise fit and well can be safely discharged home providing that chest radiography and ECG show no abnormality. Careful implementation of this policy has reduced unnecessary burdens in an already busy cardiothoracic surgical unit. 
Use of audit improve to accident and emergency senior house officers' assessment of functional anatomy of the hand H Guly, D Boon, P Riou

Accident and Emergency Department, Frenchay Hospital, Bristol

Objectives-It was felt that new senior house officers (SHOs) in accident and emergency $(A \& E)$ are poor in their assessment of hand anatomy and function. This audit was undertaken to assess this level of knowledge and to reassess after intervention in the form of a tutorial.

Method - Two investigators evaluated 32 A\&E SHOs from emergency departments in the South West region using a 30 point questionnaire on anatomy and function of the hand. Standards were set such that 22 points were considered as essential knowledge. The other eight were considered minor and were given less weighting. They were then given a tutorial with an opportunity to demonstrate their new learnt skills. They were re-examined towards the end of their posts to audit retention of knowledge.

Results-Preaudit confirmed poor performance. Mean score for the total of major and minor points was 17.7. After intervention these scores improved dramatically showing that the tutorial was successful and that knowledge was retained. Mean score after intervention was $24.5(\mathrm{p}<0.01)$. Apart from one all made an improvement. For the major points mean score before intervention was 14.6 and after was 19.3 ( $\mathrm{p}<0.05)$.

Conclusion-Examination of the hand by SHOs in A\&E is poor but with appropriate tuition this can be improved significantly.

Integration with host nation medical facilities: an evolution in military contingency medicine

D Blake, $T$ Burge

1st Medical Group, Langley AFB, Virginia, USA

The transition of military contingencies to small theatre conflicts has necessitated the simultaneous evolution of military contingency medical support. Medical assets now need to be more compact, more rapidly mobilised, and more adapted to the specific theatre of operations. Recently, medical teams from the United States and the UK were mobilised in support of Operation Desert Fox/Bolton. These teams were comprised of surgeons, anaesthetists, nurses and technicians, as well as necessary administrative support personnel. This coalition medical team was stationed at a host nation, 260 bed tertiary care facility. The mission of this team was to integrate directly with the staff of this facility and develop an operational plan to support coalition military action in the Kuwait theatre of operations. The goal of this endeavour was then to establish a possible contingency facility for use in the event of future military action in this theatre. We present our assessment of this integration, and discuss briefly its impact on overall future military operations.

Effects of surgery on plasma natriuretic peptides release in orthopaedic patients G N Onuoha, E K Alpar University of Birmingham

Atrial stretch results in myocyte release of the atrial natriuretic factor $(1-126)$ and alpha atrial natriuretic peptide 1-28 ( $\alpha$ ANP). The $N$ terminal (1-30) fragment, proatrial natriuretic peptide (proANP) is release on an equimolar basis with the $C$ terminal (99-126) active

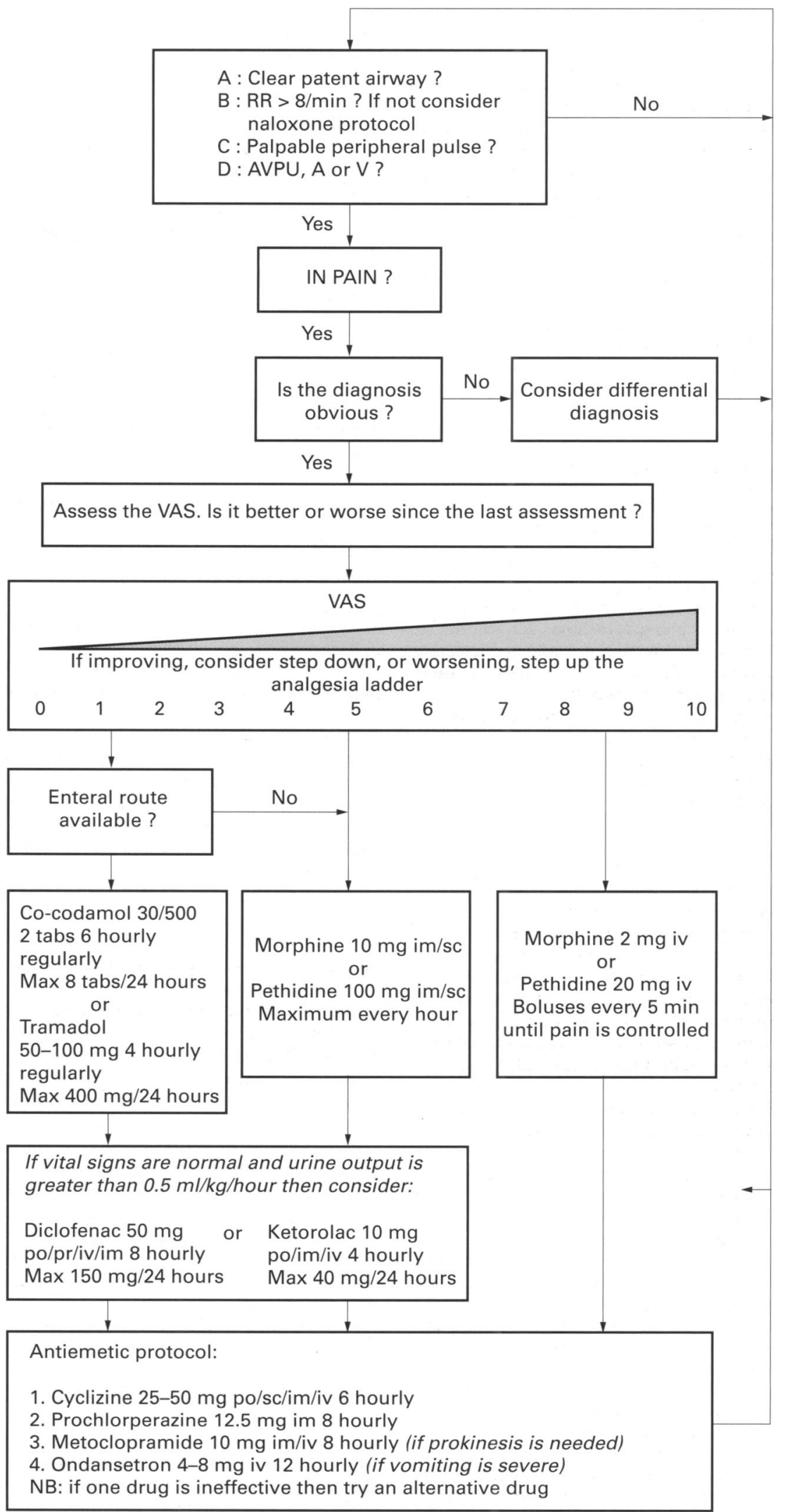

Naloxone protocol:

Dilute 400 micrograms of naxolone in $4 \mathrm{ml}$ of normal saline.

Give $1 \mathrm{ml}$ iv every minute until RR $>10 / \mathrm{min}$.

NB: Further doses may be needed due to the short half life of naxolone compared to morphine.

Figure 1 Analgesic protocol (see Hocking and DeMello on previous page). 
hormone and may be assayed simply due to in vitro stability.

This study was undertaken to evaluate the effect of surgery on the release of $\mathrm{N}$ terminal peptide of proANP and of $\alpha$ ANP in myoskeletal injured patients. Neuropeptides were sampled from 15 orthopaedic patients within 24 hours after injury and 24 hours after operation. Patients' median age was 50 years (range $24-90) ; 46.7 \%$ were men. The mean age of 17 non-orthopaedic subjects who were used as controls was 40.4 years (range 23-87).

A specific enzyme immunoassay method suitable for the measurements of plasma $\mathrm{N}$ terminal proANP and $\alpha$ ANP was used. The natriuretic peptides were observed to be higher in patients up to 24 hours after injury. After operation, however, proANP plasma concentrations were attenuated while $\alpha \mathrm{ANP}$ concentrations were reduced. These increased levels observed with $\mathrm{N}$ terminal proANP were $1160 \%$ greater than those obtained from $\alpha$ ANP $(p<0.05)$. Like proANP, creatine kinase (a known marker of tissue damage) and elastase (a decisive marker for postoperative inflammation/and infectious complications), were found to be higher in patients plasma samples and the increased levels were pronounced 24 hours after operation.

It was concluded that the titration of plasma $\mathrm{N}$ terminal peptide of proANP may be a useful analytical tool for therapeutic intervention during the early phase of orthopaedic injuries; it could also be a useful tool in detecting myoskeletal disturbances involving muscle damage and bone fractures resulting in surgical intervention up to 24 hours after operation.

Atiology of injuries missed in the emergency room

E Hammacher, J Meijer, M Janssens, Chr van der Werken

University Hospital Utrecht, The Netherlands

Missed injuries in trauma patients are a known problem. The reported rates of missed injuries in trauma patients during their total stay in the hospital is estimated to be between $10 \%$ and $34 \%$. Little is known about the percentage of injuries missed in the emergency department alone. We therefore conducted a retrospective study to identify the factors involved in multiply injured trauma patients in whom injuries were missed or not suspected in the emergency department.

All trauma patients seen at the emergency department in 1996 with an injury severity score (ISS) of more than 13 after initial assesment were included in the study. Each patient had initial evaluation and resuscitation in the trauma room. All findings were registered in an automated documentation system. The notes and discharge letters of the intensive care unit, the hospital or if applicable the revalidation centre, were screened for diagnoses added to the original diagnoses made at the emergency department. A injury was considered missed if it was not found or not suspected during the diagnostic work-up of primary and secondary screening in the trauma room. The patients were divided into two groups: one in which one or more injuries were missed and a group in which no injury was noted to have been missed. These two groups were studied with regard to a number of factors.

In 1996 there were 376 admissions to the emergency department of patients with an ISS of more than 13. Of these patients, 31 were transferred to other hospitals, 12 died in the emergency department, and four had insuffi- cient follow up documentation. This left 327 patients $(87.5 \%)$ with sufficient documentation for adequate assesment. Thirty seven of these 329 patients $(11.2 \%)$ had 47 injuries which were missed or not suspected in the emergency department. In 13 of these 37 patients $(35 \%)$ the discovery of the missed injury prompted delayed treatment. Significant factors in missing injuries were the ISS score of the patient, the means of referral, and the time of day the patient arrived at the emergency department.

The most important conclusion was that awareness of the fact that injuries are frequently missed is the best form of prevention. The percentage of patients with a missed injury can be used as a quality indicator.

All basic surgical trainees should rotate through an accident and emergency post $S$ Britten

Frenchay Hospital, Frenchay, Bristol

A six month post in accident and emergency (A\&E) is no longer a compulsory requirement for basic surgical training. Meanwhile, trauma teams have emerged in many UK hospitals to receive seriously injured patients, often comprising specialist registrars in anaesthesia general surgery and orthopaedics, with little involvement of basic surgical trainees. This may lead to the appointment of specialist registrars in surgical disciplines who have had little exposure to the initial management of the seriously injured. This study documents the experience of a senior house officer (SHO) undertaking a six month post in the $A \& E$ department of a district general hospital, with particular reference to experience gained in the reception of the seriously injured patient. Methods-At the time of the study, Queen Alexandra Hospital, Portsmouth saw 70000 new patients a year. Data concerning the nature of cases seen were collected retrospectively from all patient record cards signed by the author during the six month placement. Cases included in the figures that follow include all attendances in the resuscitation room, all cases of significant body cavity trauma, and all cases of significant musculoskeletal trauma seen during that time.

Results-During the period studied the author was present in the resuscitation room on 41 occasions, of which 10 episodes involved the management of a multiply injured patient. Other resuscitation room activity included 17 cardiac arrests, of which six patients left the department alive, five respiratory arrests, and nine other episodes.

The patients seen were as follows: four skull fractures, two base of skull fractures, and three traumatic intracranial bleeds; 17 spinal injuries, including two with cord injury; five significant cases of chest trauma; four of abdominal trauma; and five significan urinary tract injuries; 159 cases of fracture or significant soft tissue injury in the upper limb; and 122 such cases in the lower limb.

Discussion-The six month post in A\&E provided the basic surgical trainee with significant exposure to the care of seriously injured patients. Such a post should be compulsory to ensure that specialist registrars on appointment have already received exposure to seriously injured patients.
Involvement of a UK regional cardiothoracic unit in cardiothoracic trauma care J Atkins, J Hyde, V Barnett, K Porter ${ }^{\star}$, T Graham

Cardiothoracic Unit, Queen Elizabeth Hospital, Birmingham and ${ }^{\star}$ Multiple Injuries Unit, Selly Oak Hospital

The University Hospital Birmingham Trust has an accident and emergency unit and multiple injuries unit at Selly Oak Hospital and a cardiothoracic unit at the Queen Elizabeth Hospital, which are two miles apart. This is a retrospective review of 23 months (1 April 1997 to 28 February 1999) involvement of the cardiothoracic unit with in trust cardiothoracic trauma referrals (and two out of trust). The cardiothoracic unit runs a trauma registry on a PATS computer database of referrals and interventions. The database records patient details, consultation details, nature of injuries, cardiothoracic interventions, and outcome.

Results-There were a total of 43 patients reviewed; $33(76.5 \%)$ were male. The mean age was 35.3 years. There were seven $(16.3 \%)$ iatrogenic injuries, $17(39.5 \%)$ road traffic accidents, seven $(16.3 \%)$ penetrating injuries, $23(53.5 \%)$ with blunt trauma, $33(76.5 \%)$ had a consultant review, $21(48.8 \%)$ had operative interventions, 17 (39.5\%) intercostal drains, and $11(25.5 \%)$ deaths.

Summary-This is a review of an imperfect but typical UK practice as the cardiothoracic unit is off site from the trauma unit. These data suggest that with an almost $50 \%$ operative interventions rate versus only a $40 \%$ intercostal drain insertion rate that the cardiothoracic unit could have a larger involvement in cardiothoracic trauma care within the trust. In the future the trauma registry data should be collected with a view to an integrated trauma registry with the trauma unit. The relative high incidence of iatrogenic trauma is a reflection of the Queen Elizabeth Hospital's specialist services which perform a high rate of great vessel intravascular access.

\section{Revised Glasgow coma scale}

J Batchelor, A McGuinness, J Ryan

Department of Accident and Emergency and Department of Conflict Recovery, University College London

Objectives-The aim of the study was to identify some of the weaknesses in the adult Glasgow coma scale (GCS) with a view to developing a modified coma scale.

Method-To address some of the weakness in the GCS. (A) The GCS does not accurately define the clinical state of patients with a GCS of 15 and hence is a poor discriminator of patients with so called "minor head injuries". (B) The GCS was designed as a linear analogue scale, however because the scale begins at 3 and not 1 the definition of zero is unclear. (C) The GCS more correctly describes a conscious scale and not a coma scale. Results-The revised GCS:

- Utilises the same three components as in the conventional GCS.

- Linear scale from 1-15.

- Score 1: Head injury with concussional symptoms.

- Score 2: Head injury with persistent vomiting.

- Score 3-15: The same as the conventional GCS except in the reverse order.

Conclusion-The revised adult GCS provides an alternative linear analogue approach to the assessment of patients with head injuries. 
Pre-hospital training courses-time to stop teaching chest drain insertion? R Steyn, C Cheeseman

West Midlands CARE Team, Selly Oak Hospital, Birmingham

Background-Chest injuries are the primary cause in $25 \%$ of trauma deaths and are implicated in an additional $25 \%$. Of these $25 \%$ may be preventable. Trauma teaching emphasises the importance of the "golden hour" which starts from the time of injury. Prehospital trauma courses teach chest drain insertion. Chest drain insertion is an important cause of iatrogenic chest injury in hospital. Prehospital insertion in less than ideal circumstances is even more difficult and has a greater risk.

Method-The West Midlands CARE Team comprises doctors and nurses specially trained and equipped for pre-hospital care and are tasked by the ambulance service to attend the more serious incidents. Most of the doctors hold the Diploma in Immediate Medical Care and all are well versed in chest drain insertion. All patients treated by the West Midlands CARE Team between 1 September 1995 and 31 October 1997 were reviewed.

Results-1356 patients were treated (road traffic accident 461 (34\%), other trauma 353 (26\%), medical $542(40 \%))$. Thirty had chest injuries (11 penetrating, 19 blunt trauma). Eleven needle thoracocentesis were performed. No patients required pre-hospital intercostal drainage. Pneumothoraces were present in seven with blunt trauma, one had a tension pneumothorax.

Conclusion-Intercostal drainage is infrequently required pre-hospital. Most doctors attending pre-hospital training courses have rarely inserted chest drains and hence are unlikely to retain the skills required. Doctors with appropriate experience should not be restricted in inserting chest drains prehospital, but intercostal drainage should not be a routine component of basic level pre-hospital training courses.

The SIrUS Project-towards a determination of which weapons cause "superfluous iniury and unnecessary suffering" S J Mannion, Robin M Coupland International Committee of the Red Cross, Geneva

The 1977 protocol I addition to the Geneva Conventions specifies that "it is prohibited to employ weapons, projectiles and material and methods of warfare of a nature to cause superfluous injury and unnecessary suffering". Although the use of such weapons has long been illegal under international law, there has been no widely accepted definition of what constitutes "superfluous injury and unnecessary suffering".

The SIrUS project is an attempt to bring objectivity to the above concept, using the scientifically measureable injuries caused by legitimate weapons as a baseline. The data for this baseline were derived from the database of the International Committee of the Red Cross (ICRC), which documents the wounds of over 28000 war injured victims. In order to determine the collective effects of conventional weapons the database was analysed looking specifically at the parameters of mortality, proportion of large wounds (ICRC classification), duration of hospital stay, number of operations required, transfusion requirements, central compared with peripheral injuries, and permanent disability in survivors.
By reference to the baseline defined above four objective criteria are proposed in an attempt to define a weapon that causes "superfluous injury and unnecessary suffering". If the weapon results in:

(1) Specific disease, specific abnormal physical or psychological state, specific and permanent disability or disfigurement.

(2) Field mortality of more than $25 \%$ and hospital mortality of more than $5 \%$.

(3) Grade 3 wounds as measured by the Red Cross wound classification system.

(4) Effects for which there is no recognised and proved treatment.

The medical and related professions have a unique responsibility for the treatment of the victims of war. The SIrUS project does not purport to make a moral judgment relating to the use of weapons in war but does attempt to establish objective criteria upon which weapons systems can be judged and then international law applied.

\section{Evaluation of border crossing helicopter trauma care}

P Groenendijk, S Rödel, A van Walsum, P Vierhout

Department of Surgery, Medical Spectrum Twente, Enschede, The Netherlands

Since 1985 there has been cooperation between two German trauma centres and our hospital in the Netherlands concerning the emergency treatment of trauma patients. An area of $7850 \mathrm{~km}^{2}$ is covered by a border crossing system of helicopter trauma care.

Every year approximately 1000 flights are carried out by a German helicopter service. After triage at the accident scene approximately 100 patients are transported to one of the three hospitals by helicopter. We evaluated the patients admitted in our hospital in the years 1995, 1996, and 1997.

There were 56 patients, 40 male and 16 female, with a mean age of 31 years (range 4-64). Cause of trauma was a traffic accident in 46 patients, a fall from height in seven, a sports injury in three, and a suicide attempt by gunshot in one patient. All patients were stabilised by an emergency physician at scene. Fifty three patients were intubated and ventilated, eight patients received a thorax drain, and two patients were primarily resuscitated. Mean time between announcement and arrival of the patient in our hospital was $33 \mathrm{~min}$ utes.

Forty nine patients were (haemodynamically) stable at admission. Forty six admissions concerned a neurotrauma, 26 a thoracic trauma, 25 a severe injury of the extremities, and eight an abdominal trauma. Twenty nine of the 56 patients required urgent surgery. Intervention surgery because of haemodynamic instability was carried out in seven patients; this was succesful in four patients.

Overall 10 of the 56 patients died in our hospital, six on the day of admission, two after two days, one after three days, and one after six days. For the patients who died the mean injury severity score (ISS) was 32 (range 20-66), for the surviving patients the mean ISS was 22 (range 4-59). For all patients the mean ISS was 22 (range 4-66). Duration of admission at the intensive care unit varied between 0 and 79 days.

Approximately $18 \%$ of the trauma patients in our large border crossing region presented and were admitted to our hospital. Mean time between announcement and arrival was half a hour. Altogether $18 \%$ of the patients died in our hospital. Retrospectively, we were not able to define the revised trauma score for all patients. Therefore, registration of all trauma patient data is now carried out by a computer program designed for the Dutch Association for Traumatology.

TRISS methodology; a flawed mathematical model

J Batchelor, M Gavalas, A McGuinness, J Ryan

Department of Accident and Emergency and Department of Conflict Recovery, University College London

Objectives - The aim of the study was to review some of the weaknesses in the TRISS methodology and to identify some new concepts upon which trauma scoring models might be based.

Methods-The revised trauma score (RTS) has an inherent weakness in that it uses the same arithmetic scale for each of its three components despite the fact that the three components measure different ranges of physiological response. This results in bias with head injuries being over-scored and shock states being under-scored.

Results-Elimination of bias from the RTS may partly be achieved by defining four physiological states-(1) minor, (2) moderate, (3) severe, and (4) decompensated-for the three components of the RTS and scoring each response equally.

Conclusion-This study has defined a four level physiological response model which may enable a more effective mathematical model to be applied to trauma scoring.

Pre-hospital management of burn patients: time for some coordination

\section{K Allison}

This study was commissioned on behalf of the Faculty of Pre-hospital Care at the Royal College of Surgeons of Edinburgh

The aim of the study was to assess the current management of burn patients by the UK ambulance services, given the increasing importance placed on clinical governance and advances in pre-hospital care, specifically analgesia and dressings.

Each of the UK ambulance services was contacted via a postal questionnaire and given approximately two months to reply; one reminder was sent to non-responders.

Eight simple questions related to the provision of burn care were asked.

- 31 replies were received from a total of 39 questionnaires (79\% response)

- $10(32 \%)$ services said that they had a treatment policy for burns patients, six (19\%) said that they followed IHCD/BASIC ambulance guidelines, and $18(58 \%)$ said that they had no treatment policy.

- $30(97 \%)$ services sent patients to their nearest accident and emergency department.

- Seven (23\%) services had a local bypass policy for burn patients.

- $26(84 \%)$ services employed cooling and 14 $(45 \%)$ used dressings as basic first aid.

- 12 different types of dressing were used for burn patients.

- Analgesia prescribed for burn patients included nalbuphine hydrochloride (23 or $74 \%$ ), Entonox (30 or $97 \%$ ), Calpol (one or $3 \%$ ), diamorphine (two or $6 \%$ ), and tramadol (three or $10 \%$ ).

- $23(74 \%)$ services gave oxygen to all burn patients and $28(90 \%)$ cannulated patients, with or without fluid administration. 
There seems to be a wide variation in the basic approach to the first aid and pre-hospital care of burns patients. We feel that it is time to get a standard approach across all ambulance services for this large group of patients. We propose to coordinate this through the Faculty of Pre-hospital Care and with liaison with burns units and ambulance services, leading to a position statement for the pre-hospital management of patients with burn injuries.

\section{Recent advances in military trauma J Navein, J Ryan MIM/USUHS}

Combat casualty care is fundamentally different from trauma care in the civilian setting. Civilian trauma care is based on the Advanced Trauma Life Support (ATLS) programme which does not translate readily to the operational environment. Nor does it match the skills, resources, or isolation of a medic managing a casualty in a hostile environment. An extensive and multispecialty evidence base has developed to underpin ATLS and guide the civilian trauma team; the same has not been true of the military.

Casualty care at the point of wounding is considerably more complex than is generally understood. The operational environmen impacts directly on care of the casualty and casualties also directly impact on the operation. Similarly, deployed hospitals need to adapt to changing tactics. Modern conflicts are highly mobile and large, relatively immobile hospitals are no longer appropriate. Surgical doctrine must adapt accordingly, but where is the experience, let alone evidence, to support such changes?

In 1992, trauma programmes for Special Forces medics were developed in the UK and US. Workshops followed which began to "internationalise" the process, adding expertise from Israel, Russia, and France. The International Committee of the Red Cross (ICRC) and military programmes developed the surgical management of war wounded and the process culminated in joint military/ICRC conferences on the management of war wounded in 1998. This paper describes the process to date.

\section{Poster presentations}

Clinical evaluation of the "respimeter": a new disposable respiratory rate counter A Breakell, C Townsend Rose

Department of Accident and Emergency Medicine, Royal Liverpool University Hospital, Liverpool

Objectives-To clinically evaluate a new, disposable mask mounted respiratory rate counter for use in the emergency setting.

Methods-Random visits to a local acciden and emergency (A\&E) department were made and all patients wearing oxygen masks were recruited into the study. The sensor was attached to the outside of the mask. One researcher auscultated the chest (blinded), the other counted the sensor activity. Respiration was counted over one minute. A total of 40 patients were recruited into the study. If the sensor recorded an error of more than two breaths compared with auscultation, this was recorded as a sensor failure.

Results-The respiratory rates of 40 patients were measured. There were 28 males and 12 females. Twenty six patients were wearing Hudson $100 \%$, 14 a System 22 mask. Over one minute the sensor gave a perfect score in
28 cases $(70 \%)$. It was accurate to within one breath in 37 cases (93\%), to within two breaths in $98 \%$ of cases, and in one case the sensor failed.

Conclusion-The sensor is inexpensive, disposable, accurate, and attaches to any currently available oxygen mask. The sensor is unaffected by patient position, mask type, and oxygen flow. The sensor has a digital counter and can be used to monitor breathing in many clinical situations, for example pre-hospital, in the $A \& E$ department, in disaster and war zones, in in-flight care, and in military and civilian shipping.

\section{Advanced transfer bridge $S$ Robbins \\ Queen's Hospital, Burton on Trent}

With constant transfer of critical patients, the need to develop and provide a safe and accurate system that transfers patients is vital. A present most intensive care and accident and emergency units do not have a standardised procedure. Most of the vital equipmen required for the journey is placed upon the trolley sides or by other unacceptable means.

Personally I have many years' experience in transfer and, with the help of a local company, I have devised a complete safe way of transferring patients to and from hospitals. I have decided to call this new way of transferring patients the advanced transfer bridge (ATB) system. It comprises a fitted frame that carries the equipment over the patient's legs. On top there is enough room for monitoring, ventilation, infusion pumps, suction, and oxygen Through the centre of the frame is a cradle to allow a " $D$ " cylinder of oxygen for transferring from ambulance to the required unit.

There are several advantages of the ATB system: a clear system of monitoring observa tion for all staff during the transfer, a safe and secure method of securing monitoring and ventilation equipment, and the facility of having two or more infusions pumps at any on time. All these benefits of a new ATB system would standardise patient safety at all times, resulting in a smooth and safe transfer.

It is estimated that 10000 patients are transported each year between hospitals. ${ }^{1}$

1 Parke TJ, Rimmer ME. Monitoring the critically 1997;13:150-4.

Innovation in the realm of trauma coordination, clinical supervision, education, and development

A Hutchings

Southampton University Hospitals NHS Trust

Several different models of trauma coordination can be found in the UK. Some follow American models while others have developed to meet local needs. This paper outlines a model aiming to improve the standards of care experienced by patients/families and reduce the stress and anxiety experienced by practitioners after accident and emergency care.

The nature of traumatic injuries requires the multidisciplinary team to have a broad knowledge base of the principles of treatmen and an appreciation of the multiplicity of injury (Synder 1993). The psychosocial impact of injury on the patient and their family may be complex requiring expert knowledge and ability to support people through a stressful event.

As a specialist practitioner I am concerned that we should provide an environment in which the health and well being of staff and the quality of care provided to patients and families is of the highest possible standard.

This paper explores the model we have introduced over the last two years. It describes the support and clinical advice available throughout the trust as well as the clinical supervision programme within trauma and orthopaedics. It explains the key role in trauma management planning, patient preparation for surgery, and multiprofessional communication. It goes on to identify the role in the provision of seamless care for patients nursed in a number of care settings by several different teams, clinical expertise in arranging complex transfers from other centres, and the need to explore and solve problems encountered within the system.

Patterns of emergency referrals to a regional plastic surgical unit

A Hazari, F Schonauer, $\mathrm{K}$ Anderson, J Pereira, H Belcher

Department of Plastic Surgery, Queen Victoria Hospital, East Grinstead, West Sussex

Acute referrals comprise $40 \%$ of the work of a plastic surgery unit. The Queen Victoria Hospital is the regional centre for burns, plastic and hand surgery for the South East Thames region covering Kent, Sussex, and East Surrey with a catchment population of 3.5 million. The on-call senior house officer takes emergency referrals in plastic surgery over the telephone from accident and emergency departments, general practitioners, and other specialist units. The aim of this study was to quantify the percentage distribution of the various plastic surgical subspecialty emergencies and their outcome. An additional aim was to examine the organisation of the referral system and identify its strengths and drawbacks.

Data were collected over a period of three months from September to November 1998, and involved accurate documentation on a "trauma card" of the date and time of the call, the referring hospital, clinical details, and advice given. Hand injuries that fulfilled pre-set criteria were booked for the day surgery trauma list operational the next morning. The management outcome in each patient was recorded.

There were 684 referrals originating from 41 sources, with the 12 main referring hospitals providing $68 \%$ of the workload Hand injuries accounted for $54 \%$ of the cases, followed by burns (24\%), facial injuries $(13 \%)$, and lower limb trauma $(8 \%)$. The management resulted in $61 \%$ of the referrals being admitted of which $46 \%$ underwent surgery.

This system of referral was found to be easy, effective, and suitable for the geographical area of south east England and is likely to be applicable to other similar units.

Surgery on plasma myosin heavy chain fragments of skeletal muscle

G N Onuoha, E K Alpar, M Laprade*, B Dean, D Rama ${ }^{\star}$, B Pau $^{\star}$

University of Birmingham and *Sanofi Recherche, Montpellier, France

Serum concentrations of myosin heavy chain (MHC) were determined serially in 38 orthopaedic patients using a competitive radioimmunoassay to $\beta$-type MHC fragments. Fourteen of these patients were surgically treated (pre/post-op) and the others did not receive any operation (OP) within the two day study period. These were compared with 16 nonorthopaedic control subjects. The time course 
Table 1 Results: mean (range)

\begin{tabular}{lllll}
\hline & Age & MHC $(\mu U / l)$ & $C K(I U / l)$ & Myoglobin $(\mu g / l)$ \\
\hline OP day 1 & $62.9(17-80)$ & $93.3(0.5-566)$ & $230.3(19-850)$ & $90.1(23.6-237)$ \\
OP day 2 & & $250.4(27.5-690)$ & $463.4(72-2769)$ & $140.4(27.6-738)$ \\
Pre-op & $58.2(24-94)$ & $99.3(2.3-471.1)$ & $122.1(20-711)$ & $74.8(17.2-310.6)$ \\
Post-op & & $273.2(6.5-1202)$ & $294.4(20-1324)$ & $118.0(10.2-406.9)$ \\
Control & $47.8(23-70)$ & $72.9(11.9-173)$ & $97.2(19-203)$ & $32.0(16-85.2)$ \\
\hline
\end{tabular}

of the myosin concentrations are typical and monophasic for all patients. The values for the kinetic parameters of myosin release in these orthopaedic patients are comparable with those previously reported in patients with myocardial infarction. We also measured cardiac troponin-I (cTnI) release in all subjects. cTnI was negligible in all samples measured in the cTnI assay; this therefore excludes a protein release from the heart muscle (cardiac $\beta$-type MHC) of these subjects. We further made attempts to compare the release of this contractile protein to other known markers of tissue damage within 48 hours from the onset of injury. Results are shown in table 1 .

Bone fractures result in a biphasic myoglobin release profile, delayed creatine kinase (CK) peaks and late MHC fragment concentrations of slow skeletal muscle myosin. These findings also provide evidence for an injury of slow twitch skeletal muscle fibres in response to orthopaedic injuries and surgery. From a practical point of view, we observed that early injury does not seem to qualitatively upset myosin kinetics. Furthermore this results indicate that myosin release is a quantitative index for tissue damage especially in late or postoperative diagnoses. It was finally concluded that the titration of serum myosin was probably of little clinical value for therapeutic intervention during the early phase of orthopaedic injuries; it could, however, be a useful tool in detecting any myoskeletal disturbances involving muscle injury or bone fractures resulting in membrane leakage of myoskeletal cells in a retrospective basis, postoperative analysis, or as a prognostic tool.

\section{Fracture/surgery on skeletal troponin-I} release

G N Onuoha, E K Alpar

University of Birmingham

The skeletal isoform of troponin-I (sTnI) is a myofibrillar protein highly specific for myoskeletal injury and for identifying the origin (cardiac or skeletal) of muscular damage. We use a recently developed new generation immunoassay with high analytical sensitivity to measure sTnI in orthopaedic patients, before and after operation. We hypothesised that sTnI could detect and monitor muscular damage in patients with bone fracture.

Plasma from 32 individuals (17 healthy controls, 15 orthopaedic patients undergoing operative repairs) were assessed for skeletal/ cardiac troponin-I (scTnI) using a two step immunoenzymometric assay method-one being specific for cardiac troponin-I isoform (cTnI). Creatine kinase (CK), myoglobin, and elastase were measured using the ELISA method. Results are shown in table 2.
cTnI was non-detectable in all subjects in the cardiac troponin-I assay (attributing results to the skeletal isoform of troponin-I). CK and myoglobin were higher in patients and the difference in release of elastase (compared with control subjects) was statistically nonspecific.

These data provide evidence for identifying the origin (skeletal) of the muscular damage such as found in bone fractured man. In addition, the combined use of scTnI assays appears suitable for providing clinicians in sports or orthopaedic medicine new biological markers of high relevance and reliability for the diagnosis of skeletal and muscle specific injuries.

\section{Photographic wound documentation} after open fracture

M Solan, J Calder, C Gibbons

Princess Royal Hospital, Haywards Heath and University of Sussex

Objective-To assess the ability of accident and emergency (A\&E) departments to photograph an open fracture.

Design-Telephone questionnaire of $51 \mathrm{~A} \& \mathrm{E}$ departments.

Main outcome measures-Availability of a fully functioning camera at the time of the survey. Access of the department to a camera 24 hours a day.

Results $-41 \%$ of all departments surveyed were unable to take a photograph of an open wound at the time of the survey. Of those able to offer a photographic service a further 10 departments $(20 \%)$ had this facility only during normal "daylight" hours. The cause of the inability to photograph wounds was lack of film in 12 hospitals $(23 \%)$, theft in three hospitals $(6 \%)$, breakage in four hospitals $(8 \%)$, and was unexplained in two hospitals (4\%).

Conclusion-Open fractures require early assessment and meticulous treatment if devastating complications are to be avoided. The British Orthopaedic Association/British Association of Plastic Surgeons guidelines advise photographing an open wound. This removes the need for repeated inspection and reduces infection rates. Our study found that $61 \%$ of A\&E departments did not have 24 hour a day facility to photograph the wound over an open fracture.

\section{The pelvic stabilisation belt-a cadaveric} study

M Solan, L Ripley, M Lavelle

Princess Royal Hospital, Haywards Heath and University of Sussex

Rotationally unstable pelvic ring injuries account for nearly one third of all pelvic ring disruptions. Open book (external rotation) injuries in particular are often associated with

Table 2 Results: median (range) extreme, blood loss. In haemodynamically unstable cases reduction should be achieved in the resuscitation room. External fixation is widely used for this purpose.

This cadaveric study demonstrates that unstable external rotation injuries can be significantly reduced by bringing the patient's legs together and completely closed using a non-invasive pelvic belt placed over the greater trochanters.

The pelvic belt could be used instead of external fixation for haemorrhage control and patient transfer. It can be applied extremely quickly with no need for other equipment or special expertise, and may be particularly useful if there is no immediate orthopaedic cover.

As a method for pelvic reduction and temporary stabilisation this specially designed device has advantages over both other noninvasive techniques and external fixation.

\section{Review and suggestions for management} of minor head injuries

S West, D Shewring, P Evans

East Glamorgan Hospital, Church Village, Near Pontypridd

Objectives-To review the aetiology of head injury. The Glasgow coma scale (GCS), relation to alcohol consumption, and self discharge rate among patients admitted for minor head injury in the Cardiff Royal Infirmary was recorded.

Methods-A basic questionnaire was designed to include all the study parameters. This was completed at the time of admission. All patients admitted with a minor head injury over an 11 week period from mid-October 1997 to January 1998 were included.

Results - There were 45 admissions for head injury during the study period. Of these $4.5 \%$ were related to sport, $8.9 \%$ to road traffic accidents, $24.5 \%$ to falls in the home, $26.6 \%$ were intoxicated with alcohol but not related to assault, and $35.5 \%$ followed alleged assaults. Overall, we found $60 \%$ of admissions were intoxicated with alcohol. Admissions rose steadily through the week. Alcohol concentrations followed a similar pattern. In all cases the GCS was never lower than 14 Only one case required further investigation after admission. Associated injuries were trivial in all but two cases. Self discharge was not found to be common, but those who did had either been assaulted or were intoxicated with alcohol. Irrespective of the aetiology of admission no patients stayed longer than 18 hours.

Conclusions-We feel the results support the use of short stay wards for the management of minor head injuries. The use of such wards may reduce the burden on main hospital wards.

Ambulance work: emotional impact

D A Alexander, S Klein

Medical School, Aberdeen

Among health care workers ambulance personnel are among the most likely to retire early on health grounds. Aetiological factors have been sought but no study has explored the impact of "critical incidents" on mental welfare.

Aims-Funded by the Scottish Office this survey identified: (a) the levels of "burnout" and general and post-traumatic symptoms; (b) which incidents are the most emotionally disturbing, and (c) which factors attenuate their effects.

\begin{tabular}{lllll}
\hline Pre-op & $18.2(14-42.3)$ & $65(20-711)$ & $46.4(17.2-310.6)$ & $96.9(0-346.8)$ \\
Post-op & $21.4(15.2-75.2)$ & $131(20-1324)$ & $67.4(10.2-406.9)$ & $96.9(86.7-520)$ \\
Control & $2.22(1.5-4.97)$ & $112(21-203)$ & $25.7(5.9-135.5)$ & $96.9(15.3-163)$ \\
\hline
\end{tabular}


Method-A cross sectional survey by questionnaire and interview of ambulance personne who provide accident and emergency cover. Results-Univariate and multivariate analyses confirmed that among these staff:

(1) Despite high levels of job satisfaction they displayed high levels of "burnout" and high levels of general and post-traumatic symptoms.

(2) Exposure to road traffic accidents and medical emergencies, particularly those involving children, severe injury and inadequate briefing, have deleterious effects that may be durable and cumulative.

(3) Personality, peer support, and certain coping methods attenuate these effects.

Conclusions-It is concluded that:

(1) Repeated exposure to emotionally disturbing events compromises the emotional welfare of ambulance personnel.

(2) Job satisfaction is not a valid barometer of emotional well being.

(3) The ambulance service must address selection, training, welfare provision, and managerial practices.

\section{Ocular trauma: psychiatric sequelae D Alexander, R Kemp, S Klein, J Forrester Medical School, University of Aberdeen}

Our understanding of the impact of trauma has derived mainly from the annals of military combat and major catastrophes. Recently, efforts have been made to identify the effects of daily trauma including assault, motor vehicle accidents, and industrial accidents. The subject of ocular trauma has been a neglected area of research. This study aims to: (i) describe the prevalence of post-traumatic psychopathology and problems of adjustment following ocular trauma and (ii) to identify those factors that are predictive of such sequelae.

Method-This casenote and interview study included approximately 40 patients admitted to the ophthalmology ward of Aberdeen Royal Infirmary. Data were collected from: (i) structured interview, (ii) standardised measures of psychopathology and social adjustment, and (iii) theatre records and hospital computer database.

Results-All patients were male, aged between 18 and 65 years. Injuries included penetrating, blunt, chemical and thermal ones, $55 \%$ of which were as a result of industrial injury. The level of psychiatric "caseness" was identified at $45 \%$. By univariate and multivariate analyses, factors which related to this level of psychopathology and other problems of adjustment were identified.

\section{Conclusions-It is concluded that:}

(1) Ocular trauma is strongly related to psychopathology and problems of adjustment.

(2) It is possible to identify factors relating to poor prognosis and adjustment.

(3) There are opportunities for early psychiatric intervention. Acknowledgements: Thanks are due to the charity,
Remedi, for financial support and $\mathrm{Mr} \mathrm{A}$ Scott (SR) and $\mathrm{Dr} M$ Costen (SHO) of Aberdeen Royal Infirmary.

Carpal tunnel decompression during butress plating of the distal radius-is it necessary?

O Odumala, C Ayekoloye, G Packer

Southend Hospital NHS Trust, Essex

The objective of this study was to evaluate the effectiveness of prophylactic carpal tunnel decompression in preventing median nerve dysfunction during buttress plating of the distal radius.

A comparative study of 69 unselective consecutive patients with distal radial fractures managed by volar plating between 1995 and 1998. Patient's clinical notes were assessed for clinical parameters of median nerve dysfunction and all patients followed up for a minimum of six months. Twenty four patients had prophylactic carpal tunnel decompression and 45 patients had not.

The study population consisted of 42 females and 27 males and mean (SD) age was 56 (19.9) years. Overall 17 patients (25\%) developed median nerve dysfunction postoperatively. Nine patients had formal prophylactic carpal tunnel decompression and eigh patients had not; this was not statistically significant $(p=0.07)$. In addition prophylactically decompressed patients had more than twice the relative risk $(2.1$; confidence interval 0.94 to 4.74 ) of developing median nerve dysfunction. All cases resolved spontaneously except for three cases that required carpal tunnel decompression of which two patients were not prophylactically decompressed.

We conclude that median dysfunction does not alter the cause of median nerve dysfunction and may increase postoperative morbidity.

Management of minor head injuries by non-specialists

N Buxton, H Pau, M Cartmill

Department of Neurosurgery, University Hospital, Nottingham

Objective-To assess the nature and outcome of patients admitted with a minor head injury (MHI) to a hospital containing a regional neurosurgical unit.

Design-A retrospective analysis of the admissions to this hospital in 1996 with a diagnosis of $\mathrm{MHI}$

Subjects-All patients aged 16 years or over admitted with a diagnosis of $\mathrm{MHI}$, defined as Glasgow coma score $>12$; a total of 618 fulfilled this criteria of whom $89(14.4 \%)$ were referred to the regional neurosurgical unit (male:female 63:26; 70.8\%:29.2\%).

Results-The reason for referral was: no accident and emergency beds in 47 (53\%), not stated in $22(25 \%)$, "social reasons" in four $(4 \%)$, and in two $(2.25 \%)$ because they had been under a neurosurgeon some years previously for totally unrelated conditions. Only two of $24(8.3 \%)$ patients who had computed tomography during their admission had anything abnormal detected, neither of whom needed any intervention beyond simple observation. There was one late complication, a seizure five months after the MHI.

Conclusion-It is our contention that no MHI need be admitted under the care of neurosurgeons in this country and that patients with MHI who do need specialist neurosurgical input can be identified by neuro-observations in a non-specialist setting and referred for advice or action accordingly.

Transfer of the critically ill; a three year prospective survey

M Sanders, S Cockroft

Intensive Care Unit, Salisbury District Hospital, Wiltshire

Over a three year period we have prospectively collated data relating to the interhospital transfer of intensive care unit (ITU) and high dependency unit (HDU) patients from Salisbury District Hospital.

In addition to demographic data, transfer timings, destination, diagnosis, and data relating to patient support were collected. Any critical incidents were also recorded.

During this period a total of 86 transfers to other hospitals were conducted. The transfer was conducted for specialist treatment in $70 \%$ of instances and in $30 \%$ the transfer was necessary due to excessive demands upon the local ITU facilities. Average distance travelled was 35.6 miles (range 25-123)

From acceptance by the receiving hospital, a mean delay of 100 minutes occurred before the transfer started (range 5-450). In $72 \%$ of transfers the patients required full ventilatory support. In $43 \%$ of transfers, the accompanying physician was of senior house officer grade whereas only $17 \%$ were accompanied by a consultant. Critical incidents occurred during $26 \%$ of the transfers.

We conclude that the interhospital transfer of ITU and HDU patients is a complex task that requires adequate equipment provision and medical expertise and training to avoid potential patient morbidity and mortality. Continual audit is essential to ensure that the standards recommended by the Intensive Care Society in "Guidelines for the transport of the critically ill adult" are met.

Use of helicopters in the Peak District Mountain Rescue Organisation

James Williams, Adam Brooks

Peak District Mountain Rescue Organisation

The Peak District was the first national park in the UK, established in 1951. Seventeen million people live within 60 miles of its boundary. With 20 million people visiting each year it is the second most commonly visited national park in the world.

The Peak District Mountain Rescue Organisation (PDMRO) consists of seven individual teams. In 1998 they were involved in 134 incidents. This included 59 hill walking incidents, ( $75 \%$ of which had an injury to the leg or ankle), 33 "non-mountain" incidents (mainly searches for missing people), 23 rock climbing accidents, three hang gliding/paragliding accidents, seven mountain bike/trail bike accidents, and three sheep rescues! There were six fatalities.

The PDMRO can use either the RAF Sea King helicopter from Leconfield or police helicopters from Manchester or Sheffield to assist in incidents. They are used for casualty evacuation, for searches, or for deployment of mountain rescue team personnel onto the hillside.

We have analysed the use of helicopters by the PDMRO in 1998 to determine the appropriateness of the individual helicopters to specific mountain rescue team tasks and to review the criteria used for their deployment.

Helicopters were used on 33 occasions ( $25 \%$ of incidents), with the police helicopter deployed on $76 \%$ of these, mainly for searches and the RAF Sea King on $24 \%$ of occasions, predominately for the evacuation of injured casualties.

Helicopters are very useful in the mountain rescue environment, but the appropriateness of individual helicopters for specific mountain rescue team tasks must be recognised in the call out criteria. 